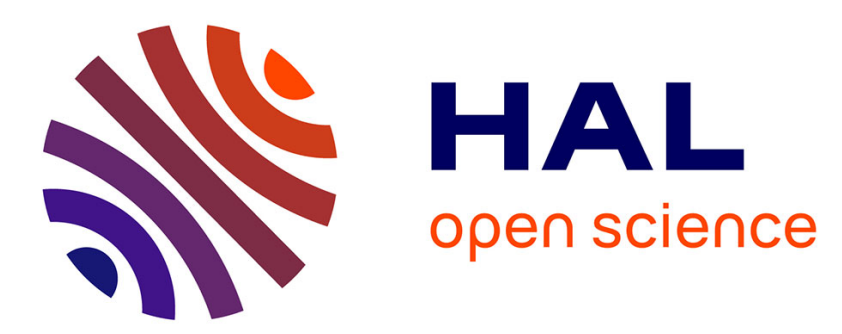

\title{
Effet de la disponibilité en nitrate sur le comportement de jeunes pêchers en culture hydroponique
}

\author{
Robert Habib, Bernard Leboeuf
}

\section{To cite this version:}

Robert Habib, Bernard Leboeuf. Effet de la disponibilité en nitrate sur le comportement de jeunes pêchers en culture hydroponique. Agronomie, 1987, 7 (2), pp.101-109. hal-00884974

\section{HAL Id: hal-00884974 \\ https://hal.science/hal-00884974}

Submitted on 1 Jan 1987

HAL is a multi-disciplinary open access archive for the deposit and dissemination of scientific research documents, whether they are published or not. The documents may come from teaching and research institutions in France or abroad, or from public or private research centers.
L'archive ouverte pluridisciplinaire HAL, est destinée au dépôt et à la diffusion de documents scientifiques de niveau recherche, publiés ou non, émanant des établissements d'enseignement et de recherche français ou étrangers, des laboratoires publics ou privés. 


\title{
Effet de la disponibilité en nitrate sur le comporte- ment de jeunes pêchers en culture hydroponique
}

\author{
Robert HABIB \\ avec la collaboration technique de Bernard LEBOEUF \\ I.N.R.A. Station d'Agronomie - Domaine Saint-Paul-Centre de Recherches d'Avignon, F-84140 Montfavet
}

Mots clés additionnels : Prunus persica $L$. Batsch, teneur en azote, réserves azotées, matière sèche.

Young peach trees «Suncrest/GF 655-2 » cultivated hydroponically were given three different nitrate feeds. During the time of exponential increase of dry matter, two to four trees per feed were taken at different times and their roots, trunks, shoots and leaves cut up and analysed for their dry matter $(\mathrm{g})$ and nitrogen content ( $\left.\mathrm{g} . \mathrm{g}^{-1} \mathrm{DM}\right)$. The results were interpreted by analysis of variance. The limited nitrate feeds did not induce changes in dry matter increase. However, the nitrogen content and the total nitrogen amount obtained by calculation were modified but proportionally less than expected from the theoretical difference between the three treatments. This can be interpreted as a modification in nitrate absorption per unit root. The results are discussed in relation to a nitrogen transfer model in the tree presented in another paper.

Additional key words : Prunus persica (L.) Batsch, growth, content, nitrogen reserve, dry matter.

\section{INTRODUCTION}

La nutrition azotée d'une plante supérieure cultivée peut être présentée schématiquement en 4 étapes :

- la fourniture minérale (fertilisation, minéralisation, nitrification),

- l'approvisionnement qui correspond à la mise à disponibilité des éléments nutritifs aux racines par le transfert des solutés dans le sol ou la croissance du système racinaire,

- l'absorption qui se rapporte à la pénétration des éléments minéraux dans la plante (dans le cas de l'azote, essentiellement $\mathrm{NO}_{3}^{-}$),

- l'assimilation métabolique (de notre point de vue, principalement la réduction du nitrate et la répartition des assimilats).

Les arbres fruitiers sont en outre caractérisés par l'existence d'un pool de réserves azotées endogènes dont l'importance pour leur développement est maintenant bien établie (OLAND, 1959 ; TAYLOR, 1967 ; TAYLOR \& MAY, 1967 ; STASSEN et al., 1981 ; TROMP, 1983). Le stockage l'année $\mathrm{n}$ de ces réserves et leur mobilisation l'année $\mathrm{n}+1$ (ou $\mathrm{n}+\mathrm{i}$ ) peuvent être considérés comme un mécanisme de fourniture retardé de l'azote sous une forme directement utilisable pour la croissance et le développement des arbres.

Il existe une littérature abondante concernant l'étude des réserves azotées (voir par exemple la synthèse bibliographique de TITUS \& KANG, 1982). Mais il 
existe à notre connaissance peu de tentatives, exceptées bibliographiques, de synthétiser ces connaissances en un schéma de fonctionnement le plus simple possible, qui puisse rendre compte à la fois de la pénétration de l'azote dans l'arbre (sous forme nitrique) et de sa répartition ultérieure dans les différents organes en croissance, concurremment à l'utilisation de l'azote endogène de réserve pré-existant. Le but de ce travail est de fournir la base expérimentale nécessaire à l'élaboration et à la mise à l'épreuve d'un modèle de la dynamique de répartition de l'azote endogène chez un jeune arbre fruitier. Ce modèle devrait être basé sur les relations de fonctionnement dynamique entre les organes végétaux d'un arbre (pour simplifier: racines, tronc, rameaux, feuilles). Aussi, notre objectif est de mesurer simultanément un certain nombre de variables sur la plante entière, dont on peut penser, a priori, qu'elles interviennent dans la dynamique de répartition de l'azote. Il est également de tester la sensibilité de ces relations à certaines modifications du milieu, en particulier la disponibilité du nitrate.

Il était a priori peu raisonnable, dans une $1^{\text {re }}$ étude, de tenter de prendre en compte les 4 étapes de la nutrition azotée évoquées précédemment, ainsi d'ailleurs que de vouloir analyser les transferts d'azote organique en différenciant les formes dites soluble (principalement les acides aminés et amides) et insoluble (principalement les protéines). Aussi nous nous sommes volontairement limité :

$1^{\circ}$ à une culture des arbres en solution hydroponique stricte : les aspects concernant la fourniture minérale et l'approvisionnement se trouvent, de fait, éliminés,

$2^{\circ}$ à un dosage de l'azote total, en considérant que le nitrate absorbé était immédiatement réduit sous forme organique dans le système racinaire, bien que cette hypothèse soit en partie infirmée par d'autres travaux (données non publiées).

\section{MATÉRIELS ET MÉTHODES}

\section{A. Matériels}

Les pêchers que nous avons utilisés, «Suncrest/GF 655-2 ", étaient des scions de 1 an. Le porte-greffe avait été choisi pour sa vigueur moyenne, compte tenu des conditions de culture qui étaient envisagées. Avant « plantation » les arbres ont été fortement rabattus :

- au niveau racinaire, en ne laissant subsister que le pivot et le minimum de racines devant assurer la reprise (de l'ordre de 0,5 à $1,5 \mathrm{~g}$ en matière sèche),

- au niveau aérien, afin de ne permettre le débourrement que de 2 à 3 bourgeons à bois par arbre.

Cela nous a permis de considérer que la majorité des racines observées dans la suite de l'essai était nouvellement formée et de maintenir le développement aérien des arbres dans des limites compatibles avec nos moyens expérimentaux (renouvellement des solutions nutritives, préparation des échantillons végétaux), tout en limitant l'hétérogénéité inter-arbres quant aux parties aériennes.

A la plantation (avril 1983), le poids frais des scions après élagage variait de 90 à 260 grammes (moyenne :
137 grammes, écart-type : 26 grammes). On constate là une variabilité, inhérente au matériel végétal, des conditions initiales. Ceci est l'un des principaux problèmes liés à l'expérimentation sur arbres fruitiers, y compris en conditions contrôlées.

La composition de la solution nutritive de base, exprimée en meq. $1^{-1}$, était : $\mathrm{Ca}^{++}(2), \mathrm{K}^{+}$(2), $\mathrm{Mg}^{++}(1,5), \mathrm{Na}^{+}(0,7), \mathrm{Cl}^{-}(2), \mathrm{SO}^{-}-\overline{4}(3,5), \mathrm{PO}_{4} \mathrm{H}_{2}^{-}$ $(0,7)$. Elle comprenait, en outre, des oligoéléments et du séquestrène. Elle était complétée par $\mathrm{Ca}^{++}$ $\left(10 \mathrm{meq} \cdot 1^{-1}\right)$ et $\mathrm{NO}_{3}^{-}\left(10 \mathrm{meq} \cdot 1^{-1}\right)$, la fréquence des apports nitriques permettant de différencier les traitements (cf. § II B). Le pH initial de la solution variait de 5,4 à 5,5 , il n'a pas été corrigé entre 2 renouvellements de solution. En pleine végétation, celle-ci était renouvelée tous les 7 à 14 jours à raison de 5,5 litres par pot. Entre 2 renouvellements de solution nutritive un réajustement par de l'eau déminéralisée pouvait être réalisé en fonction de la demande climatique. La solution était aérée par un «bullage » continu d'air comprimé. Il n'a pas été procédé à un contrôle de la microflore, pouvant entrer en compétition avec les racines pour les éléments minéraux, afin de ne pas modifier les capacités d'absorption des systèmes racinaires.

\section{B. Méthodes}

\section{Traitements « azote »}

Trois traitements ont été différenciés par la durée de contact entre la solution nutritive contenant du nitrate et les systèmes racinaires. Sur la base de la semaine (7 jours) ces traitements ont été définis comme suit :

- traitement dit « T7 » : apport du nitrate lors du renouvellement de solution (durée du contact nitrateracines : 7 jours) ;

- traitement dit «T5 » : apport du nitrate 2 jours après le renouvellement de solution (durée du contact nitrate-racines : 5 jours) ;

- traitement dit «T3» : apport du nitrate 4 jours après le renouvellement de solution (durée du contact nitrate-racines : 3 jours). Ces apports ont été réalisés à masse constante de $55 \mathrm{~m}$ moles de $\mathrm{NO}_{3}^{-}$.

Dans certains cas, deux semaines séparaient deux renouvellements de solution. Les durées de contact ont alors été adaptées sur la base de 14 jours (T5 : 10/14 jours ; T3 : 6/14 jours). Par ailleurs, il s'est écoulé 38 jours entre la plantation et le premier renouvellement de solution. Dans ce cas, les durées de contact nitrate-racines ont été :

$$
\begin{aligned}
& \text { T7 : } 38 / 38 \text { jours, } \\
& \text { T5 : } 34 / 38 \text { jours, } \\
& \text { T3 : } 30 / 38 \text { jours. }
\end{aligned}
$$

Les arbres ont été répartis de façon aléatoire sur 3 « blocs », c'est-à-dire 3 lignes de plantation orientées Nord-Sud, comprenant chacun 4 《placettes» de 10 arbres contigus. Les lignes de plantation étaient protégées du vent dominant par une haie brise-vent orientée Est-Ouest. Chaque placette correspondait à l'un des 3 traitements "azote »; soit un total de 4 placettes, 40 arbres, par traitement sur l'ensemble des 3 blocs. Sur ces 40 arbres, seule la moitié environ devait, a priori, être prélevée : nous avons doublé la taille du dispositif car les techniques d'élagage utilisées 
pour "homogénéiser » les scions à la plantation (cf. $\S$ II A) pouvaient être considérées comme traumatisantes. L'absence de variabilité inter-bloc à la plantation a été vérifiée par analyse de variance sur les poids frais initiaux des arbres.

\section{Matériel végétal}

A intervalles de temps déterminés, il devait être prélevé, au hasard, un arbre par bloc et par traitement pour analyse ; soit un total de 3 arbres par traitement pour chaque date de prélèvement. Dans certains cas cependant des erreurs de manipulation nous ont conduit à en prélever 2 ou 4 . Il a été réalisé 6 prélèvements, le premier 38 jours et le dernier 108 jours après la plantation. Cette période coïncide avec la phase de croissance exponentielle en matière sèche. Les prélèvements d'arbres ont toujours été effectués à l'occasion d'un renouvellement de solution. Il a été prélevé 52 arbres sur l'ensemble de l'essai.

Chaque arbre a été fractionné en ses différentes parties anatomiques :

- racines de diamètre inférieur à $2 \mathrm{~mm}$,

- tronc du porte-greffe (tronc PG),

- tronc du cultivar (tronc $\mathrm{CV}$ ),

- rameaux de l'année,

- feuilles,

- « déchets » comprenant le point de greffage et le pivot racinaire.

Dans l'examen des résultats, il sera uniquement fait mention du tronc «total » qui regroupe alors le tronc du porte-greffe, celui du cultivar et les " déchets".

Le nombre de rameaux et de feuilles par arbre, ainsi que la longueur de ces rameaux ont également été relevés.

Après pesée en frais, le matériel végétal a été stocké à $-20^{\circ} \mathrm{C}$ en attente du séchage qui a été réalisé par lyophilisation. On a vérifié que les échantillons lyophilisés perdaient moins de 2 p. 100 de leurs poids après 48 heures à l'étuve $105^{\circ} \mathrm{C}$. Après lyophilisation, les échantillons étaient conservés à la température ambiante.

\section{Mesures}

\section{a) Nitrate en solution:}

Lors de chaque renouvellement de solution, il a été réalisé un dosage du nitrate restant en solution pour les arbres devant être prélevés. La mesure a été réalisée par ionométrie ou colorimétrie (CIESIELSKI et al., 1978). Par ailleurs, la consommation hydrique a été estimée par pesée de la solution non absorbée.

b) Matière sèche :

La matière sèche après lyophilisation a été pesée pour chacune des fractions anatomiques citées au paragraphe II B 2 .

\section{c) Teneur en azote :}

Les échantillons ayant été broyés en totalité, le dosage de la teneur en azote ( $\mathrm{g}$ de $\mathrm{N}$ pour $100 \mathrm{~g}$ de $\mathrm{MS}$ ) des échantillons de racines, tronc $P G$, tronc $C V$, rameaux et feuilles, a été réalisé, après minéralisation sulfosalicilique à chaud d'une partie aliquote du broyat $(0,1$ à $0,5 \mathrm{~g})$, par colorimétrie (auto-analyseur «Technicon ») du bleu d'indo-phénol produit par la réaction de Berthelot en milieu alcalin. La teneur en azote du tronc « total » a été déduite par calcul de celles des troncs $\mathrm{PG}$ et $\mathrm{CV}$.

\section{Analyse des résultats}

L'analyse des résultats expérimentaux a été réalisée par analyse de variance multivariate d'un dispositif non orthogonal (programme ANVARM de la programmathèque AMANCE 81 , BACHACOU et al., 1981).

Le modèle d'analyse de variance qui a été retenu est :

Valeur
$\begin{gathered}\text { observée } \\ \text { Effet } \\ \text { générale }\end{gathered}+$ bloc
Nbre de rameaux
traitement

C'est-à-dire que l'analyse a été réalisée selon un modèle en blocs aléatoires. La structure physique de l'essai correspond, en fait, à un modèle d'analyse en parcelles divisées (ceci pour des raisons d'ordre pratique), mais les hypothèses sous-jacentes à ce plan d'expérience ne pouvaient être retenues dans le cadre de cet essai, ce qui a été vérifié.

L'effet «poids initial » a été introduit dans l'analyse de variance après discrétisation en 5 classes de cette variable continue.

L'effet « nombre de rameaux » est lié à l'élagage des arbres. Celui-ci a conduit au développement de 2 (34 arbres) ou 3 (16 arbres) rameaux par arbre. On a également observé un arbre avec un seul rameau (associé au groupe 2 rameaux) et un arbre avec quatre rameaux (associé au groupe 3 rameaux).

Les résultats des tests de l'analyse de variance seront présentés sur un tableau croisant les variables analysées et les différents effets où $* *, *$, NS signifient respectivement que le test est significatif à 1 p. 100,5 p. 100 ou non significatif.

Nous ne procéderons pas à des comparaisons multiples de moyennes car les effets que nous testons correspondent en fait à des variables quantitatives continues dont la discrétisation est en partie arbitraire : c'est la pente générale du phénomène qui a un sens plutôt que le caractère différent ou non de 2 niveaux « contigus» d'un même effet. Cette remarque est particulièrement évidente en ce qui concerne l'effet « date ».

Par ailleurs, dans certains cas, nous chercherons à interpréter l'aspect multivariate de l'analyse, en particulier la matrice de corrélation des résidus du modèle d'analyse de variance. Cette matrice fournit des renseignements sur les corrélations résiduelles entre les variables analysées qui subsistent après prise en compte des effets et interaction par le modèle d'analyse de variance.

\section{RÉSULTATS ET INTERPRÉTATIONS}

L'examen du tableau I montre que l'effet " date " est toujours significatif, quelle que soit la variable envisagée. Ceci n'est bien sûr pas surprenant concernant l'analyse d'organes végétaux en croissance. Par 


\section{TABLEAU}

Résultats des analyses de variance effectuées par groupe de variables.

(i) $R A$ : racines, $T R$ : tronc, $R M$ : rameaux, $F:$ feuilles, $L G T:$ longueur totale, $L G M:$ longueur moyenne, NBT : nombre total, NBCM : nombre par $\mathrm{cm}$ de rameaux.

(ii) NS: non significatif, *: significatif au seuil 5 p. 100, **: significatif au seuil 1 p. 100.

Results of analysis of variance.

(i) $R A$ : roots, $T R:$ trunk, $R M:$ shoots, $F:$ leaves, $L G T:$ total length, LGM: mean length, NBT: total number, NBCM : number per cm of shoots.

(ii) NS : non significant, ${ }^{*}$ : significant at $5 \%$ level, ${ }^{* *}$ : significant at $1 \%$ level.

\begin{tabular}{|c|c|c|c|c|c|c|c|c|c|c|c|c|c|c|c|c|c|c|c|}
\hline \multirow{3}{*}{ Effets analysés } & \multirow{2}{*}{\multicolumn{5}{|c|}{ Matières sèches }} & \multirow{2}{*}{\multicolumn{4}{|c|}{ Teneur }} & \multicolumn{8}{|c|}{ Données phénologiques } & \multirow{2}{*}{\multicolumn{2}{|c|}{ Absorption nitrique }} \\
\hline & & & & & & & & & & & Aasse & de $N$ & & Ran & aux & Feu & illes & & \\
\hline & $\begin{array}{l}\text { (i) } \\
\text { (ii) }\end{array}$ & RA & $\begin{array}{l}\text { TR } \\
(\mathrm{g}\end{array}$ & RM & $\mathrm{F}$ & RA & $\begin{array}{l}\text { TR } \\
\text { (p. } 100\end{array}$ & $\begin{array}{l}\text { RM } \\
0 \text { MS) }\end{array}$ & $F$ & RA & TR & RM & $\mathrm{F}$ & LGT & LGM & $\begin{array}{l}\text { NBT } \\
\qquad(\mathrm{cm}\end{array}$ & $\begin{array}{l}\text { NBCM } \\
-1)\end{array}$ & $\begin{array}{c}\text { Vitesse } \\
\left(\mathrm{mg} . \mathrm{j}^{-1}\right)\end{array}$ & $\begin{array}{l}\text { Vitesse unitaire } \\
\left(\mathrm{mg} \cdot \mathrm{j}^{-1} \cdot \mathrm{g}^{-1}\right)\end{array}$ \\
\hline \multicolumn{2}{|l|}{ Bloc } & NS & NS & NS & NS & NS & NS & $*$ & NS & NS & NS & NS & $*$ & NS & NS & NS & NS & NS & NS \\
\hline \multicolumn{2}{|l|}{ «Azote » } & NS & NS & $*$ & NS & $*$ & $* *$ & NS & $* *$ & NS & $* *$ & $* *$ & $* *$ & NS & NS & NS & NS & NS & NS \\
\hline \multicolumn{2}{|l|}{ Date } & $* *$ & $* *$ & $* *$ & $* *$ & $* *$ & $*$ & $* *$ & $* *$ & $* *$ & $* *$ & $* *$ & $* *$ & $* *$ & $* *$ & $* *$ & $* *$ & $* *$ & $* *$ \\
\hline \multicolumn{2}{|l|}{ Poids initial } & NS & $* *$ & NS & NS & NS & NS & $*$ & NS & NS & $* *$ & NS & NS & NS & NS & NS & NS & NS & NS \\
\hline \multicolumn{2}{|l|}{ Nombre de rameaux } & NS & NS & NS & NS & NS & NS & NS & NS & NS & NS & NS & NS & $* *$ & $* *$ & NS & NS & NS & NS \\
\hline \multicolumn{2}{|l|}{ "Date $\times$ Azote" } & NS & NS & $* *$ & NS & NS & NS & $* *$ & $* *$ & NS & NS & $* *$ & $*$ & NS & NS & NS & NS & $* *$ & NS \\
\hline
\end{tabular}

contre l'effet «azote» n'est pas systématiquement significatif (ou non significatif) et l'interprétation en sera développée dans la suite du texte. On remarque dans un certain nombre de cas (cf. les variables des groupes «Teneur » et « Masse de N" du tabl. 1) que l'interaction « date $\times$ azote " n'est pas significative alors que les 2 effets principaux le sont. Ceci dénote un manque de puissance du dispositif pour l'analyse de cette interaction: les arbres devant être considérés comme homogènes à la plantation (à la variabilité indîviduelle près), l'interaction "date $\times$ azote " existe toujours sur le plan théorique dans ce cas particulier. Par ailleurs, on a vérifié dans certains cas (groupes de variables "matières sèches " et "masse de $\mathrm{N}$ » du tabl. 1) que les variances résiduelles n'augmentaient pas au cours du temps. Dans la suite du texte, les résultats du tableau 1 sont illustrés par des figures présentant une seule courbe moyenne ajustée quand l'effet "date " est seul significatif, ou 3 courbes ajustées quand les effets " date » et " azote " (ou l'interaction) le sont.

\section{A. Croissance des arbres}

\section{Matière sèche}

Les résultats des tests de l'analyse de variance sont présentés sur le tableau 1. La figure 1 montre l'évolution des matières sèches pour les 4 organes en valeurs moyennes ajustées. La limitation de la fourniture nitrique ne se traduit pas par une croissance significativement différente des organes végétaux excepté en ce qui concerne les rameaux (tabl. 1) pour lesquels le traitement T5 apparaît comme plus favorable. Dans ce dernier cas, l'examen de la figure 1c et l'analyse de l'interaction « dates $\times$ traitements » indique que cet effet est lié aux matières sèches rameaux mesurés lors de la dernière date de prélèvement et n'est pas systématique. Il est difficile de considérer que le traitement « $\mathrm{T} 7$ », interprété uniquement relativement à l'apport de $\mathrm{NO}_{3}^{-}$, limite la croissance des rameaux, car on ne peut retenir l'hypothèse de toxicité, compte tenu de la concentration en nitrate utilisée. Par contre, cet effet pourrait être lié à un déficit d'alimentation en potassium : dans

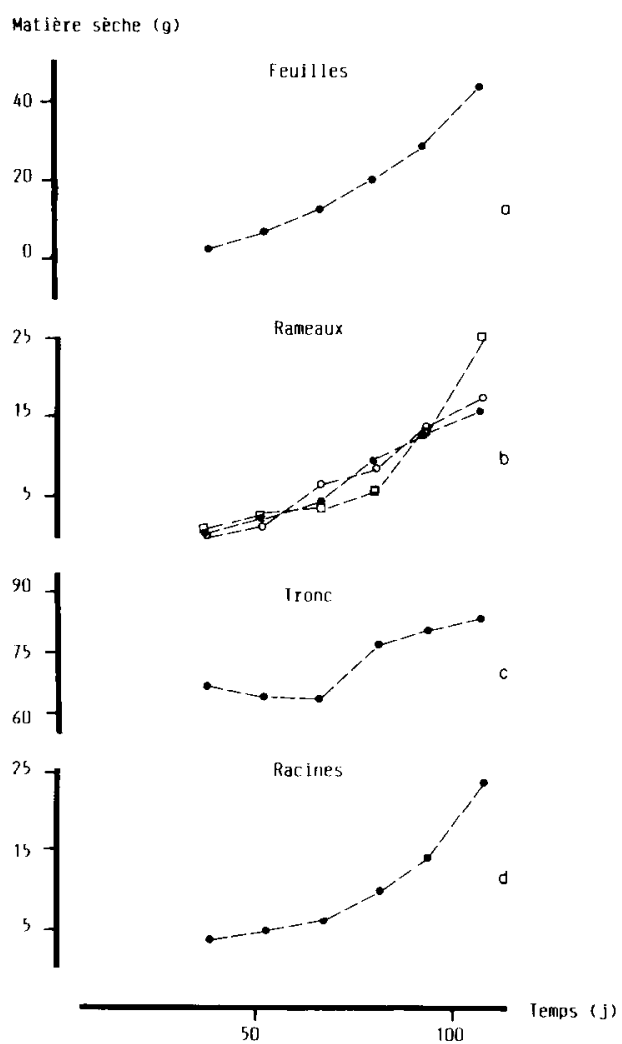

Figure 1

Croissance en matière sèche ( $g$ ).

a) Feuilles ; b) Rameaux ; c) Tronc ; d) Racines.

- :T7, $\square: T 5, \quad 0: T 3$.

Increase in dry matter (g).

a) Leaves ; b) Shoots ; c) Trunk ; d) Roots.

le traitement « $\mathrm{T} 7$ » le rapport équivalentaire $\mathrm{Ca} / \mathrm{K}$ est égal à 6 dès le renouvellement de solution et pourrait être défavorable à l'absorption de $\mathrm{K}^{+}$; dans le traitement " T5 ", il n'atteint cette valeur que 2 jours plus tard (cf. $\S$ II A et II B 1). Cette différence pourrait expliquer, qu'en période de forte « demande », le traitement « T5 » apparaisse comme plus favorable à la croissance des rameaux. Mais la vérification expérimentale (dosage du potassium) n'en a pas été réalisée. 
Par contre le traitement « T3 » ne serait pas déficitaire en $\mathrm{K}^{+}$mais effectivement en $\mathrm{NO}_{3}^{-}$.

L'effet « date » indique une croissance en matière sèche significative pour tous les organes. En ce qui concerne le tronc, cette croissance est décalée dans le temps : on constate que la matière sèche du tronc reste constante pendant environ 70 jours ( $3^{\mathrm{e}}$ prélèvement), de l'ordre de 65 grammes, puis croît d'environ 20 grammes en 40 jours.

La comparaison des courbes de matière sèche des feuilles et rameaux montre une forte analogie entre ces 2 organes qui se traduit (fig. 1a et b) par la similitude des formes de courbe. Cette liaison rend compte de liaisons fonctionnelles entre ces organes qui lient la production de matière sèche foliaire à celle des rameaux. Une relation du même ordre, apparaît à la comparaison des courbes de croissance des racines et feuilles (ou rameaux). Par ailleurs, l'analyse de la matrice de corrélation des résidus ( $\$$ II $\mathrm{B} 4$ ) indique des corrélations entre organes (feuilles-rameaux : $\mathbf{R}=0,73$; feuilles-racines : $\mathbf{R}=0,63)$ non prises en compte par le modèle d'analyse de variance, c'est-àdire qui ne sont pas liées aux effets analysés (tabl. 1). Ces corrélations résiduelles nous permettent de dire que les liaisons entre organes déduites de l'examen de la figure 1, qui représente des points moyens, se retrouvent également au niveau des arbres considérés en tant qu'individus.

On constate donc que, pendant la période d'expérimentation, la limitation de la fourniture nitrique ne s'est pas traduite par une réduction des croissances en matières sèches (excepté le cas particulier des rameaux). Par ailleurs, l'évolution des courbes, pour les trois organes néo-formés et quel que soit le traitement considéré, est de type exponentiel.

\section{Données phénologiques}

La croissance des rameaux au cours du temps (tabl. 1) se traduit par 2 phases observables sur la figure 2. Pendant la première phase d'élongation, la matière sèche par unité de longueur reste stable, de l'ordre de $0,03 \mathrm{~g} . \mathrm{cm}^{-1}$, juste $\mathrm{t} \# 70$ jours. Pendant la seconde phase, l'élongation ralentit alors que la matière sèche par unité de longueur augmente fortement, pour atteindre lors du dernier prélèvement une valeur de l'ordre de $0,15 \mathrm{~g} . \mathrm{cm}^{-1}$, correspondant à une phase de lignification. Par ailleurs, la longueur totale

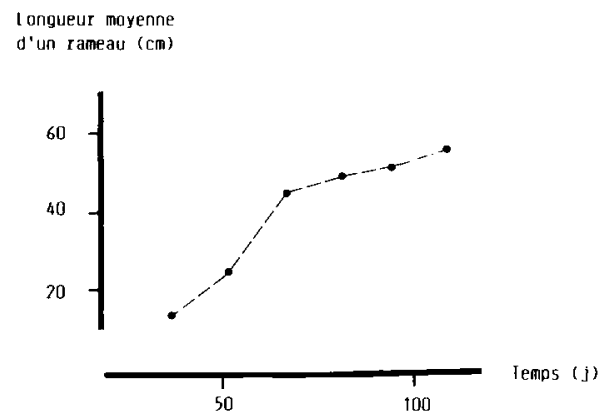

Figure 2

Croissance des rameaux $(\mathrm{cm})$.

- : T7, $\square: T 5, \quad 0: T 3$

Increase in shoot length $(\mathrm{cm})$. des rameaux est affectée par le nombre de rameaux de l'arbre (tabl. 1) : respectivement 84 et $102 \mathrm{~cm}$ (tous traitements et dates confondus) pour les cas 2 et 3 rameaux. On note cependant une compensation de la sévérité de l'élagage au niveau de la longueur moyenne des rameaux (tabl. 1) : respectivement 42 et $36 \mathrm{~cm}$ pour les cas 2 et 3 rameaux. Cet effet de compensation est encore plus marqué concernant la matière sèche pour laquelle l'effet « nombre de rameaux » n'est pas significatif (tabl. 1).

Le nombre total de feuilles par arbre n'est pas non plus affecté par le nombre de rameaux (tabl. 1). En moyenne, on observe respectivement 150 et 167 feuilles par arbre pour 2 et 3 rameaux, mais cette différence n'est pas significative. Le nombre de feuilles par centimètre de rameaux évolue linéairement au cours du temps (fig. 3) et n'est sensible ni à la limitation de la fourniture nitrique, ni au nombre de rameaux de l'arbre (tabl. 1).

Les conditions d'élagage ont donc permis de limiter la variabilité des parties aériennes (rameaux et feuilles) dans des limites qui permettent de considérer les arbres prélevés à chaque date comme représentatif du comportement moyen. Il est probable que, si la variabilité du nombre de rameaux avait été plus élevée, le comportement individuel des arbres aurait pu, par exemple, masquer l'effet de croissance que nous avons observé pour toutes les variables (tabl. 1).

\section{B. Absorption hydrique et nitrique}

Comme on le constate sur la figure 4, les 3 traitements « azote » n'ont pas donné lieu à une différenciation de l'absorption hydrique. Les matières sèches racinaire et foliaire n'ayant pas non plus été différenciées par les traitements (tabl. 1), ce résultat indique que les mécanismes d'absorption hydrique et nitrique peuvent être considérés comme indépendants dans le cadre de cet essai.

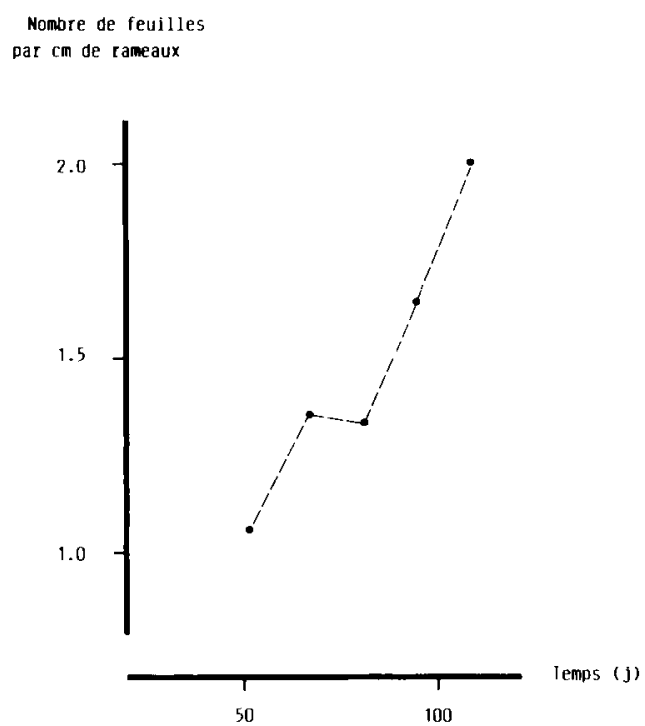

Figure 3

Nombre de feuilles par centimètre de rameaux.

- :T7, $\square: T 5, \quad 0: T 3$.

Number of leaves per centimeter of shoots. 
Absorption hydr ique $\left(1 . \mathbf{j}^{-1} \cdot\right.$ arbre $\left.^{-1}\right)$

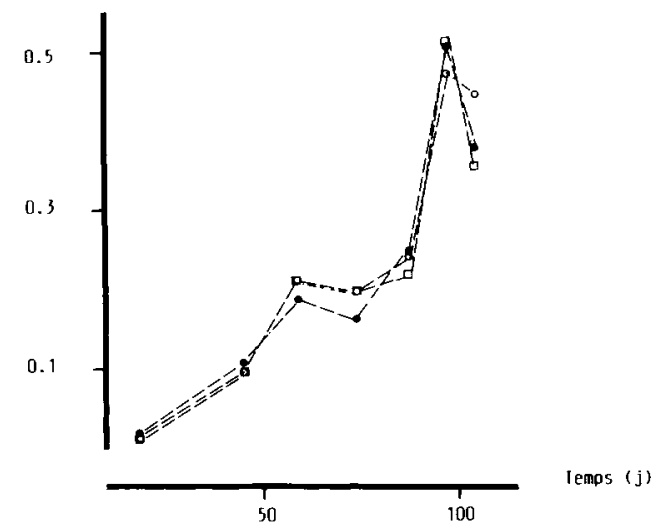

Figure 4

Absorption hydrique $\left(1 \cdot j^{-1} \cdot\right.$ arbre $\left.^{-1}\right)$.

- :T7, $\square: T 5, \quad 0: T 3$.

Water absorption (l.d $d^{-1}$. tree $\left.^{-1}\right)$.

Pour analyser les absorptions nitriques, exprimées en milligramme d'azote, nous avons ramené les valeurs mesurées d'une part sur la base de la journée pour estimer l'évolution des masses d'azote absorbées au cours du temps, et d'autre part, par jour et par gramme de racines sèches pour étudier une éventuelle modification du débit d'absorption unitaire du système racinaire. Dans les 2 cas, on constate une évolution au cours du temps (tabl. 1, fig. 5). L'absorption journalière apparente suit en moyenne une courbe croissante pour atteindre en fin d'essai des valeurs de l'ordre de $50 \mathrm{mg}$ N. $j^{-1}$ (fig. 5a). L'interaction significative (tabl. 1) est liée principalement à la différence observée entre les traitements " T3 " et " T5 » lors du dernier prélèvement (fig. 5a). La figure 5 b nous permet de dire que ces comportements (forte baisse ou hausse de l'absorption nitrique journalière) sont dus en grande partie à la variabilité individuelle des arbres, car cet effet disparaît quand l'absorption journalière est rapportée à la masse de racines (tabl. 1, fig. 5b). Le débit d'absorption unitaire des racines (fig. 5b) semble augmenter depuis la plantation pour atteindre un maximum environ un mois et demi après celle-ci, puis décroît linéairement. Cette décroissance ne peut être attribuée au vieillissement des racines qui conservent après lignification des capacités d'absorption équivalentes (ATKINSON, 1980), et nous n'avons pu l'interpréter dans le cadre de cet essai. Par contre, l'augmentation rapide des capacités d'absorption racinaire du nitrate en période de forte croissance aérienne a également été observée sur prunier (WEINBAUM et al., 1978) et serait attribuable au développement de la surface foliaire (HABIB, 1984).

La limitation de la fourniture nitrique (en terme de temps de contact nitrate-racines, cf. § II B1) ne semble donc pas se traduire par une modification de l'absorption unitaire des racines (tabl. 1, fig. 5b). Pourtant on observe une modification des teneurs en azote des organes végétaux liée aux traitements (cf. § III C). Il est alors très probable que l'absorption nitrique ait également été différenciée (pour les traitements

$$
\begin{aligned}
& \text { Absorption nitrique } \\
& \text { (mg } \mathrm{N}_{-} \mathrm{j}^{-1} \text { ) }
\end{aligned}
$$
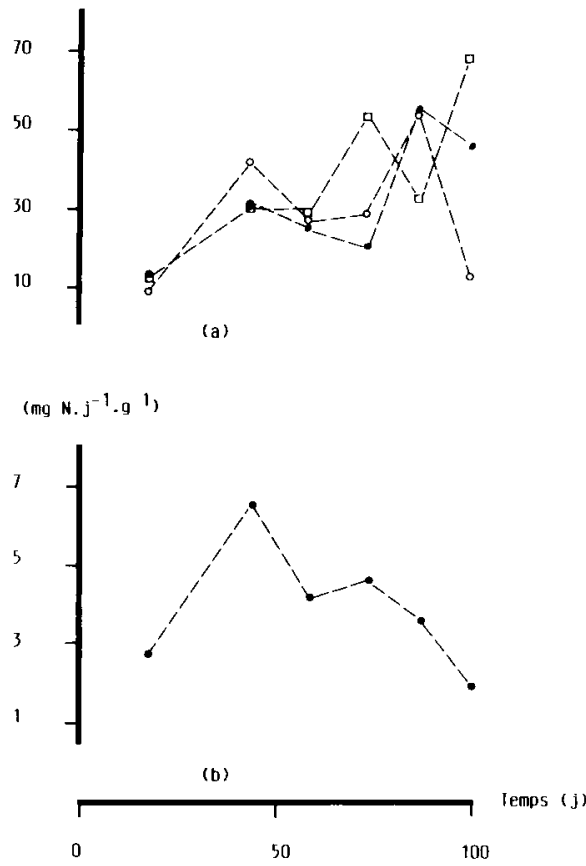

Figure 5

Absorption nitrique.

a) Vitesse d'absorption ( $m g \mathrm{~N} . \mathrm{j}^{-1}$ ).

b) Vitesse d'absorption par unité de racines (mg $N . j^{-1} \cdot g^{-1}$ )

- :T7, $\square: T 5, \quad 0: T 3$

Nitrate absorption.

a) Rate of absorption ( $m g$ N.d-1).

b) Rate of absorption per unit of roots ( $\left.m g \mathrm{~N} . \mathrm{d}^{-1} \cdot \mathrm{g}^{-1}\right)$.

«T7», « T5 », «T 3 », les vitesses moyennes d'absorption estimées sont respectivement 33,37 et $27 \mathrm{mg} \mathrm{N} . \mathrm{j}^{-1}$ ) bien que cet effet puisse être masqué par la variabilité des résultats. Ce pourrait être également lié à un manque de puissance du dispositif pour différencier les M.S. racinaires.

\section{Evolution des teneurs en azote}

L'évolution des teneurs en azote des racines, tronc, rameaux et feuilles est présentée sur la figure 6 . Il faut noter que les valeurs reproduites sont le résultat des moyennes arithmétiques des teneurs mesurées sur des parties aliquotes, elles ne représentent donc pas les teneurs moyennes réelles qui demanderaient que soient prises en compte les matières sèches de chaque organe dosé (cf. § III D). Cependant, les erreurs induites par cette méthode de calcul sont faibles, de l'ordre de quelques p. 100.

Compte tenu du manque de puissance du dispositif expérimental pour l'analyse de l'interaction " date $x$ azote » (cf. les remarques en introduction du $\S$ III), nous interpréterons, d'une part, l'évolution typique moyenne des teneurs de chaque organe, et, d'autre part, la différenciation des traitements " azote » au cours du temps, mais sans réaliser de test de comparaison de moyennes pour l'interaction.

On note (tabl. 1, fig. 6) une évolution des teneurs au 


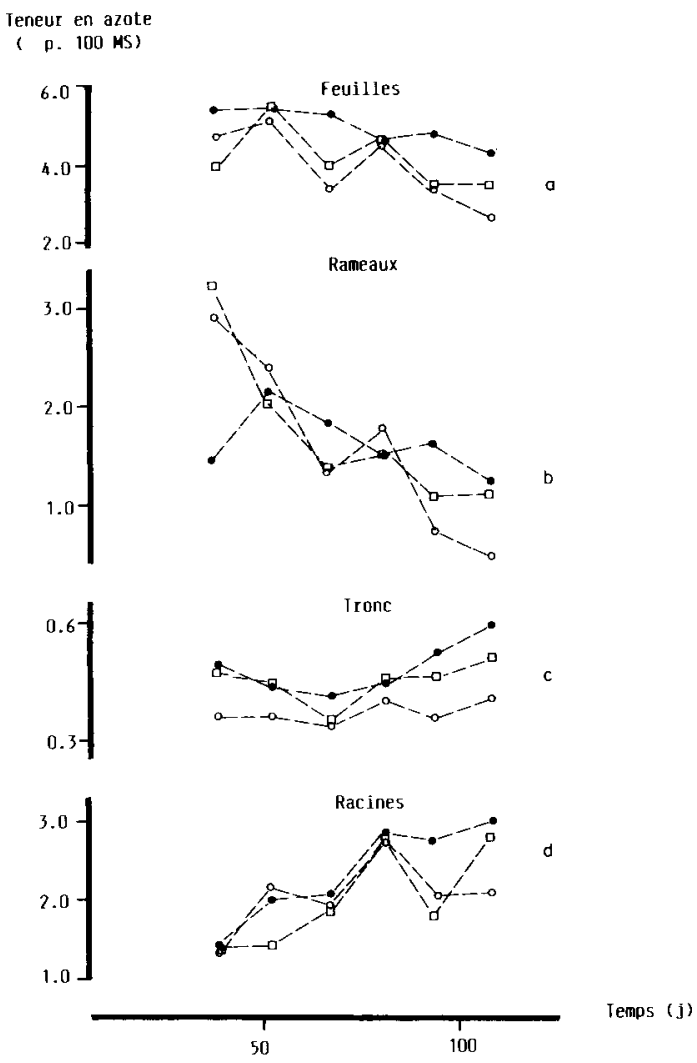

Figure 6

Evolution des teneurs en azote ( $N$ p. $100 \mathrm{MS}$ ).

a) Feuilles ; b) Rameaux ; c) Tronc ; d) Racines. - :T7, $\square: T 5, \quad \mathrm{O}: T 3$.

Changes in nitrogen content (dry matter basis). a) Leaves ; b) Shoots ; c) Trunk ; d) Roots.

cours du temps pour tous les organes analysés. Les formes typiques moyennes sont :

- pour les racines : une augmentation de la teneur (à la plantation, la teneur des racines était de l'ordre de 1 p. 100) jusqu'à un «plateau » de l'ordre de 2,5 p. 100 d'azote ;

- pour le tronc: une forme en U typique d'une phase de mobilisation puis reconstitution des réserves (teneur à la plantation de l'ordre de 0,5 p. 100, MS du tronc constante jusque $t$ \# $70 \mathrm{j}$ ). La partie porte-greffe du tronc semble reconstituer ces réserves de façon plus rapide. Cette différence est surtout notable en masse car la matière sèche "porte-greffe " est plus importante que la matière sèche «cultivar » (données non publiées) ;

- pour les rameaux : une courbe décroissante typique d'un organe en croissance (effet de dilution) ;

- pour les feuilles : une courbe décroissante également, mais de façon nettement moins accentuée que celle des rameaux, classique pour des organes foliaires en vieillissement.

Par ailleurs, on peut noter, à l'examen de la figure 6, que les teneurs moyennes sont assez bien classées vis-àvis des traitements « azote ", particulièrement pour les dernières dates de prélèvement. Pour la première date, on observe un classement parfois inverse (rameaux : fig. 6c), avec des écarts qui ne peuvent être attribués à la variabilité individuelle des arbres. Cette inversion n'a pu être interprétée dans le cadre de cet essai. Exceptée cette première date d'observation, il apparaît que la limitation de la fourniture de nitrate ne permet de différencier nettement les traitements « azote » qu'à partir de $\mathrm{t}$ \# $80 \mathrm{j}$. Et seul le traitement « T3 » conduit à une réduction sensible des teneurs en fin d'expérimentation.

La comparaison des points moyens, date par date, des figures $6 \mathrm{c}$ et $6 \mathrm{~d}$ montre une liaison entre feuilles et rameaux du même ordre que celles que nous avions notée concernant les matières sèches ( $\$$ III Al). Cependant les corrélations résiduelles ( $\$$ II B4) sont inférieures à 0,4 indiquant que l'on ne retrouve pas cette même cohérence au niveau du comportement individuel des arbres.

Enfin, l'analyse de variance a montré une hétérogénéité des teneurs en azote des rameaux, liée aux blocs (tabl. 1), les arbres du bloc médian ayant des valeurs globalement plus faibles (1,5 p. 100 MS contre 1,7 et $1,6)$, que nous n'avons pu interpréter.

\section{Evolution des maşses d'azote}

Les masses d'azote ont été obtenues par calcul : matières sèches mesurées $\times$ teneurs mesurées. L'évolution en masse d'azote des différents organes d'un arbre est un des seuls critères objectifs permettant de juger de l'augmentation (stockage) ou de la diminution (mobilisation) des réserves azotées. En effet, les teneurs sont rapportées à la matière sèche qui est constituée, entre autres, de réserves carbonées pouvant varier au même titre que les réserves azotées. Cependant la variabilité inter-arbres des matières sèches devient très importante quant à l'expression des résultats, et rend plus délicate la réalisation et l'interprétation des analyses statistiques.

L'accumulation d'azote dans les organes néo-formés, et la mobilisation puis reconstitution des réserves du tronc apparaissent nettement sur la figure 7 . La limitation de l'apport de nitrate différencie bien les masses d'azote de chaque organe, excepté les racines (tabl. 1), et plus particulièrement celles associées au traitement T3. Par exemple, les feuilles des arbres du traitement « T7 » ont accumulé en moyenne 45 p. 100 d'azote de plus que celles des arbres du traitement «T3 ». Dans le cas des racines, on avait constaté ( $\$$ III C, tabl. 1) une différenciation des teneurs liée aux traitements. Il est donc probable que la disparition de cet effet concernant les masses azotées racinaires soit causée par la variabilité propre des matières sèches des racines. L'interaction significative « date $\times$ traitement " concernant les feuilles et rameaux (tabl. 1) oppose le comportement des arbres du traitement « T5 » à ceux des traitements « T7 » et « T3 » (fig. 7c et d) lors des 2 dernières dates de prélèvement. Par ailleurs, on note que le poids initial des arbres à la plantation influe sur la masse d'azote des troncs. Cet effet n'existant pas en ce qui concerne les teneurs, on vérifie bien les résultats du même ordre constatés pour la matière sèche du tronc ( $\$$ III Al et tabl. 1).

Il apparaît donc, comme nous l'avions montré pour les teneurs, que la limitation de la fourniture de nitrate ne devient notable qu'à partir de $t \# 80 \mathrm{j}$ et que seul le traitement « $\mathrm{T} 3$ » conduit à une réduction sensible des masses azotées en fin d'expérimentation. 


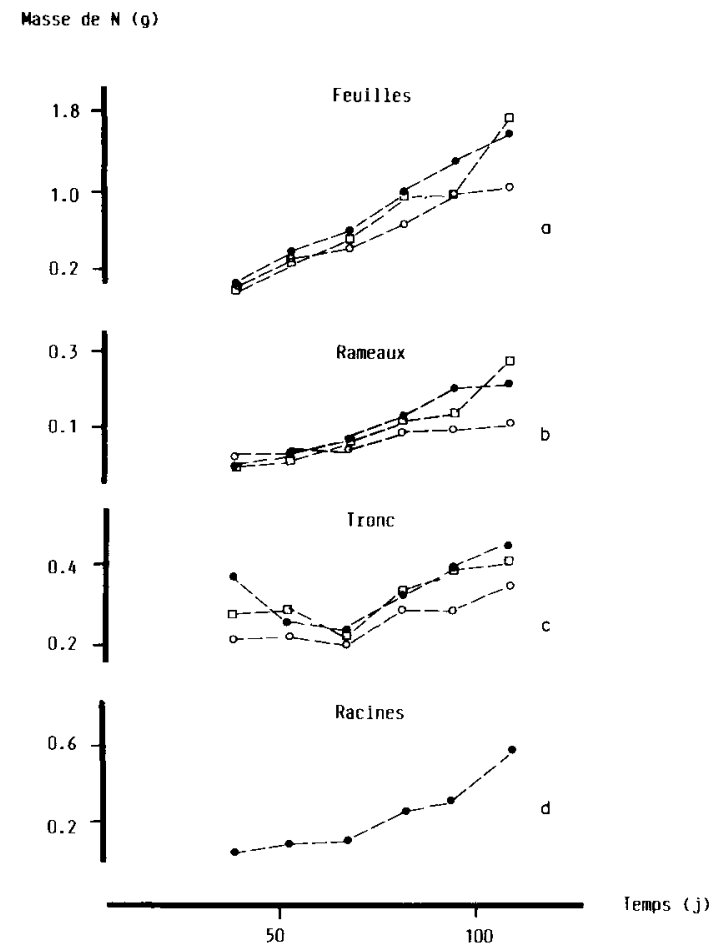

Figure 7

Evolution des masses d'azote total ( $g$ ).

a) Feuilles ; b) Rameaux ; c) Tronc ; d) Racines.

- : T7, $\quad \square: T 5, \quad 0: T 3$.

Changes in amount of total nitrogen (g).

a) Leaves ; b) Shoots ; c) Trunk ; d) Roots.

Enfin, l'analyse de variance montre une hétérogénéité des masses azotées foliaires selon les blocs : pour le bloc le moins bien protégé du vent dominant (en bordure de haie), les feuilles ont accumulé de l'ordre de $0,10 \mathrm{~g} \mathrm{~d}$ 'azote de moins que pour les 2 autres blocs. Un effet du même ordre, bien que non significatif (tabl. 1), a été trouvé pour les matières sèches foliaires. Cet effet dépressif du vent sur le fonctionnement de la surface foliaire est bien connu en arboriculture fruitière.

\section{DISCUSSION ET CONCLUSIONS}

La limitation de la fourniture nitrique telle qu'elle a été réalisée, par la diminution de la durée du contact entre le nitrate et les racines, aboutit à une baisse des teneurs et masses d'azote des organes végétaux. Mais cette baisse est moins que proportionnelle à celle que l'on pouvait attendre du fait des rapports de durée entre les traitements (cf. § II B1). Ainsi, le traitement « T7 » conduit à des absorptions nitriques (calculées sur la base des masses d'azote exportées) supérieures en fin d'essai d'environ $40 \mathrm{p} .100$ comparées à celles du traitement « T3», alors que le rapport théorique est supérieur à 2 . On obtient seulement 10 p. 100 pour le traitement « T5 ». Il y a donc eu compensation de la diminution des apports nitriques par une augmentation de l'absorption racinaire. Cette compensation pourrait en partie intervenir par le biais de la croissance des racines : celle des arbres des traitements « T5 » et «T3 » a été en moyenne supérieure de $1 \mathrm{~g}$ (matière sèche) com- parée à celle du traitement «T7 ». Mais cette différence n'est pas significative (tabl. 1). En outre, elle ne suffirait pas à expliquer la compensation observée, et il nous faut admettre que les débits d'absorption nitriques des systèmes racinaires ont également été modifiés.

Par ailleurs, nous avons montré que la limitation de la fourniture de nitrate n'avait pas significativement modifié les croissances en matière sèche. Cependant, il faut noter que les réserves du tronc ont en partie compensé la diminution des apports de nitrate en particulier pour le traitement «T3» (fig. 6b et 7b), la teneur du tronc en azote en fin d'expérimentation étant sensiblement affaiblie dans ce dernier cas.

Le début de la période de reconstitution des réserves du tronc (entre le troisième et le quatrième prélèvement) semble coïncider d'une part avec la période de croissance en matière sèche de cet organe (fig. 1b) et d'autre part avec la fin de la période d'élongation rapide des rameaux (fig. 2). Un autre essai (résultats non publiés) nous a permis de vérifier que le ralentissement puis l'arrêt de l'élongation des rameaux marquait bien le début de la croissance du tronc en masse et en volume. Cet essai a été réalisé dans des conditions de cultures différentes et avec des pêchers dont le cultivar et le porte-greffe étaient différents. Cette corrélation de croissance, et éventuellement de fonctionnement, qui paraît se traduire par un " programme " de développement, semble donc bien caractéristique de l'espèce.

En conclusion à ces remarques, il nous faut souligner le poids de la variabilité individuelle des arbŕes qui, accru par le caractère destructif des échantillonnages, conduit probablement à sous-estimer l'importance de certains phénomènes tels les corrélations de croissance ou les effets de compensation liés aux traitements ( $\S$ III A et B).

Les données expérimentales que nous avons présentées permettent en fait de décrire le comportement d'un arbre " moyen » pendant la phase de croissance exponentielle en matière sèche des racines, rameaux et feuilles. Les ordres de grandeur des variables mesurées ne sont certainement pas extrapolables à d'autres conditions de culture. Ce n'était d'ailleurs pas l'objectif de ce travail. Par contre, nous faisons l'hypothèse que les relations de fonctionnement dynamique entre les organes végétaux sont indépendantes des conditions expérimentales au moins dans leur forme, c'est-à-dire leur expression mathématique. C'est de ce point de vue que nous considérons les données recueillies comme une base expérimentale pour la mise au point d'un modèle de répartition de l'azote dans l'arbre. En effet, un certain nombre de variables ont été mesurés simultanément, et sur la plante entière, qui devraient permettre la mise au point du modèle : vitesse d'absorption racinaire du nitrate, production de matière sèche, dynamique de l'azote dans l'arbre. La modélisation envisagée devra à la fois tenir compte et rendre compte des données expérimentales que nous venons de présenter, par exemple : du point de vue de l'absorption nitrique, nous ne disposons pas d'hypothèses phénoménologiques permettant de relier l'évolution des débits unitaires d'absorption racinaire (fig. 5b) aux variables que nous avons mesurées. Il semble préférable, dans un premier temps, de considérer une valeur moyenne pour toute la 
période d'expérimentation. On pourra alors envisager un modèle (ou une fonction) d'absorption à flux constant par unité de racines. Par ailleurs, la simulation des trois traitements "azote» devra se faire avec une même fonction de production de la matière sèche (cf. $\S$ III A1), les différences entre traitements quant aux masses et teneurs en azote, devant apparaître comme liées aux seules conditions expérimentales (calendrier de fourniture nitrique).

Reçu le 9 mai 1986. Accepté le I'r novembre 1986

\section{RÉFÉRENCES BIBLIOGRAPHIQUES}

Atkinson D., 1980. The distribution and effectiveness of the roots of tree crops. Hort. Rev., 424-490.

Bachacou J., Masson J.-P., Millier C., 1981. Manuel de la programmathèque statistique AMANCE. I.N.R.A.-C.R.F. Champenoux, $516 \mathrm{p}$.

Ciesielski H., Soignet G., Catoire M., Vancayzeele P., 1978. Dosage colorimétrique des nitrates. Analusis, 6 (1), 38-44.

Habib R., 1984. La formation des réserves azotées chez les arbres fruitiers. Revue bibliographique. Fruits, 39 (10), 623-635.

Oland K., 1959. Nitrogenous reserves of apple trees. Physiol. Plant., 12, 594-648.

Stassen P. J. C., Stindt H. W., Strydom D. K., 1981. Seasonal changes in nitrogen fractions of young kakamas peach trees. Agroplantae, 13, 63-72.
Taylor .B. K., 1967. The nitrogen nutrition of the peach tree. I. - Seasonal changes in nitrogenous constituents in mature trees. Aust. J. biol. Sci., 20, 379-387.

Taylor B. K., May L. H., 1967. The nitrogen nutrition of the peach tree. Il. - Storage and mobilization of nitrogen in young trees. Aust. J. biol. Sci., 20, 389-411.

Titus J. S., Kang S. M., 1982. Nitrogen metabolism, translocation and recycling in apple trees. Hort. Rev., 4, 204-246.

Tromp J., 1983. Nutrient reserves in fruit trees, in particular carbohydrates and nitrogen. Plant Soil, 71, 401-414.

Weinbaum S. A., Merwin M. L., Muraoka T. T., 1978. Seasonal variation in nitrate uptake ef ficiency and distribution of absorbed nitrogen in non-bearing prune trees. J. Am. Soc. hortic. Sci., 103 (4), 516-519. 